\title{
Interaction of biofunctionalized gold nanoparticles with model phospholipid membranes
}

\author{
Nóra Ábrahám • Edit Csapó • Gabriella Bohus • \\ Imre Dékány
}

Received: 22 February 2014 /Revised: 17 May 2014 / Accepted: 2 June 2014

(C) Springer-Verlag Berlin Heidelberg 2014

\begin{abstract}
The understanding of the interaction of nanoparticles with cell membranes and the penetration of these nanoobjects through cell wall is highly required for their biomedical application. In this work were aimed at the study of the interaction of gold nanoparticles with model phospholipid membranes prepared at the air/water interface in a Langmuir trough. Spherical (10 and $15 \mathrm{~nm}$ mean diameter) and rod-like gold (aspect ratio: 2.8) nanoparticles were synthesized and biofunctionalized with L-cysteine and L-glutathione. The gold nanoparticles were characterized by TEM images and UVVis absorbance measurements. The interaction of the biofunctionalized gold nanoparticles with the model monolayer membrane was studied by surface pressure versus surface area compressional isotherms and by the measurement of the change in surface pressure of a preformed model membrane. The effect of the initial surface pressure of the preformed membrane was evaluated to determine the maximum insertion pressure and synergy. We have found that the driving forces of the bioconjugated $\mathrm{Au}$ nanoparticle (NP) or
\end{abstract}

Electronic supplementary material The online version of this article (doi:10.1007/s00396-014-3302-0) contains supplementary material, which is available to authorized users.

N. Ábrahám • E. Csapó · G. Bohus • I. Dékány $(\bowtie)$

MTA-SZTE Supramolecular and Nanostructured Materials Research

Group, 6720Aradi vt. 1, Szeged, Hungary

e-mail: i.dekany@chem.u-szeged.hu

N. Ábrahám

e-mail: n.abraham@chem.u-szeged.hu

E. Csapó

e-mail: tidecs2000@yahoo.co.uk

G. Bohus

e-mail: bohusgabriella@gmail.com

I. Dékány

Department of Medical Chemistry, Faculty of Medicine, University of Szeged, H720 Dóm sqr. 8, Szeged, Hungary
Au nanorod (NR) penetration into the monolayer membrane is mostly determined by electrostatic interaction and orientational van der Waals forces. Monolayer films were transferred with Langmuir-Blodgett technique onto solid substrates and the nanoparticles were visualized with AFM technique.

Keywords Nano-bio interface $\cdot$ Langmuir monolayer $\cdot$ Model membrane $\cdot$ Phospholipid $\cdot$ Gold nanoparticles $\cdot$ Biomedical application

\section{Introduction}

The study of nanomaterials towards biomedical applications is of high interest due to the novel and advantageous functional properties of these objects. Gold nanoparticles ( $\mathrm{Au} \mathrm{NPs}$ ) are one of the most widely studied candidates for such purposes, due to their biocompatibility and unique plasmonic properties [1-4]. The plasmon resonance properties of the $\mathrm{Au}$ NPs can be advantageously used in many types of therapeutic and diagnostic methods $[5,6]$, such as the so called photothermal cancer therapy, during which the cancer cells can be specifically killed with the combined application of bioconjugated Au NPs and laser illumination [7].

Large efforts are made to find new methods and improve the current techniques for biomedical purposes [8]. Choi and co-workers [9] developed a method for targeting the gold nanoparticles to hypoxic regions within tumors, which was not accessible till now. Au NPs embedded in monoyctes were used like a Trojan horse to reach these regions and exploit the therapeutic effect of them. Ba and co-workers [10] showed a method with which they are able to localize gold nanoparticles on the surface living cells while they fully preserve their capability for sensing and optothermal manipulations. Simple preparation method of plasmonic gold lace capsules with 
nanometer scale gaps between the metallic elements was presented by Yang et al. [11]. These nanostructures are interesting because SERS hot spots can be formed in these gaps, which is remarkably useful in the developement of drug delivery vehicles for therapeutic and imaging purposes or in situ monitoring of intracellular processes. For successful application of Au NPs in biological systems, Park and coworkers [12] studied the stability of the particles with a large variety of surface capping ligand and found the thiolated PEG as an advantageous material for stabilizing and for further surface modification, as well.

The transport of different molecules, drugs, nanoparticles or bioconjugated systems through living cell membranes is a key factor when developing new therapeutic agents. The understanding of the mechanism is very difficult due to the complexity of the process [13]. It was demonstrated by in vivo experiments that size, shape, surface properties of the particles (such as surface charge and the surface charge density) play important role in internalizing particles into cells $[14,15]$.

A possible way to discover the interactions of different molecules with cell membrane is to work with model membranes which mimic the living cells. Model membranes can be formed by different ways, but the most common used model systems are the liposomes, the bilayer lipid membrane and the lipid monolayer $[16,17]$.

Peetla and co-workers [18] have performed model experiments in a Langmuir trough to study the behaviour of transactivating transcriptor (TAT) peptide coated and scrambled TAT peptide coated poly(L-lactide) nanoparticles. Their experiments clearly showed the important role of the adsorbed amount and the amino acid sequence of the peptide on the particles' surface: conjugation of the nanoparticles to TATpeptide resulted in much higher rate of penetration into the membrane compared to the scrambled TAT peptide, which was explained by the different hydrophilicity of the outermost amino acids.

Considering the developing biomedical applications of gold nanoparticles, it is essential to understand their interaction with membrane surfaces, which requires proper experiments both in vivo and in vitro. In this work our main goal was to study the interaction of amino acid functionalized gold nanoparticles with model phospholipid membranes, which were formed in Langmuir trough. For the experiments, we have synthesized spherical and rod-like Au nanoparticles, and their surface was coated with biomolecules (L-cysteine, Lgluthatione). Surface pressure-surface area isotherms, time-dependent surface pressure measurements and atomic force microscopic pictures of solid supported membranes will be demonstrated and discussed for understanding the role of nanoparticle size and surface coating.

\section{Experimental}

Preparation and biofunctionalization of Au nanospheres

Spherical, monodispersed Au NPs were synthesized by the widely known Turkevich method [19, 20]. During the synthesis, the $\mathrm{AuCl}_{4}{ }^{-}$ions were reduced with trisodium citrate, the $\mathrm{Au} /$ citrate molar ratio was $1: 5$, the $\mathrm{AuCl}_{4}{ }^{-}$concentration of the dispersion was $0.2 \mathrm{mM}$ and the $\mathrm{pH}$ of the resulting sol is $6.2\left(\mathrm{HAuCl}_{4}\right.$ and trisodium citrate were purchased from Sigma Aldrich). Different sized particles were obtained with different addition rate of the reducing agent during the synthesis: smaller particles were obtained with slower addition rate.

Biofunctionalization was realized with cysteine and glutathione [21], the required amount of the coating molecule was calculated by means of the following equation:

$n_{\mathrm{cys}}=\frac{3 c V \Theta 10^{18}}{\rho_{\mathrm{Au}} r A_{\mathrm{m}} N_{\mathrm{AV}}}$

where $n_{\text {cys }}[\mathrm{mol}]$ is the necessary amount of cysteine or glutathione molecules, $c[\mathrm{mg} / \mathrm{ml}]$ and $V[\mathrm{ml}]$ are the concentration and volume of the Au nanodispersion, $\Theta$ is the surface coverage $(\Theta=1$ corresponds for monomolecular coverage), $r$ $[\mathrm{nm}]$ is the average radius of the particles determined by TEM, $\rho_{\mathrm{Au}}\left[\mathrm{g} / \mathrm{cm}^{3}\right]$ is the density of gold, $A_{\mathrm{m}}\left[\mathrm{nm}^{2} /\right.$ molecule $]$ is the cross sectional area of the molecule (for cysteine $A_{\mathrm{m}}=0.3 \mathrm{~nm}^{2}$, for glutathione $A_{\mathrm{m}}=0.8 \mathrm{~nm}^{2}[21,22]$ ) and $N_{\mathrm{AV}}$ is the Avogadro number. The required amount for $\Theta=1.2$ was applied to make sure to reach nearly monomolecular coverage.

The $\mathrm{pH}$ of the nanodispersion was adjusted to 7.4 before functionalization. The $0.01 \mathrm{M}$ solution of the biomolecules was prepared, the calculated amount of the solution was added to the $\mathrm{Au}$ NPs dispersion and stirred for further $3 \mathrm{~h}$ at room temperature. Slight change in $\mathrm{pH}$ occurred due to the addition of the biomolecules, which was readjusted to 7.4.

Preparation and biofunctionalization of Au nanorods

The Au nanorods (Au NRs) were prepared according to the seed mediated method [23]. Au seeds were prepared as follows: $7.5 \mathrm{ml} 0.1 \mathrm{M} \mathrm{CTAB}$ solution, $0.5 \mathrm{ml} 0.005 \mathrm{M} \mathrm{HAuCl}_{4}$ and $1.2 \mathrm{ml} 0.01 \mathrm{M} \mathrm{NaBH}_{4}$ solution were mixed, and the reaction mixture was vigorously shaken for $2 \mathrm{~min}$. Afterwards, it was maintained at $40{ }^{\circ} \mathrm{C}$ for $3 \mathrm{~h}$. The growth solution was prepared by mixing of $14.25 \mathrm{ml} 0.1 \mathrm{M} \mathrm{CTAB}, 0.6 \mathrm{ml}$ $0.005 \mathrm{M} \mathrm{HAuCl}_{4}, 96 \mu \mathrm{l} 0.01 \mathrm{M} \mathrm{AgNO}_{3}$ and $90 \mu \mathrm{l} 0.1 \mathrm{M}$ ascorbic acid solutions. In the last step, $20 \mu \mathrm{l}$ of the seed dispersion was added to the growth solution and was left at $40{ }^{\circ} \mathrm{C}$ for overnight. Surface capping of the Au NRs with cysteine was carried out by washing the dispersion with 
$0.01 \mathrm{M}$ cysteine solution two times. In the last step, the cysteine coated $\mathrm{Au}$ NRs were dispersed in ultrapure water and the $\mathrm{pH}$ was adjusted to 7.4.

\section{Characterization of Au NPs}

TEM images of the spherical and rod-like particles were taken on a FEI Tecnai $\mathrm{G}^{2} 20$ X-TWIN transmission electron microscope. Average size and size distribution of the particles were calculated based on the measured diameter of at least 100 particles on TEM images. UV-Vis absorbance spectra of $\mathrm{Au}$ nanodispersions were measured with Uvikon 930 spectrophotometer in a quartz cuvette.

\section{Langmuir monolayer experiments}

Monolayers of dipalmitoylphosphatidylcholine (DPPC; Sigma-Aldrich) were formed in a Kibron MicroTroughS type Langmuir trough. The lipid films at the air/water interface were characterized by surface pressure $(\pi)$ versus surface area $(A)$ isotherms. For spreading $0.1 \mathrm{mg} / \mathrm{ml}$ solution of the lipids in chloroform (Sigma-Aldrich, Chromasolv $^{\circledR}, \geq 99.9 \%$ ) was prepared. Ultrapure water or the Au nanosphere dispersions were used for subphases (0.2 $\mathrm{mM} \mathrm{Au}$ atom concentration) for isotherm measurements. After spreading of the lipids at the interface $30 \mathrm{~min}$ were left to reach equilibrium. Constant rate compression of $815 \mathrm{~mm}^{2} / \mathrm{min}$ was applied, measurements were carried out at room temperature $\left(26 \pm 1{ }^{\circ} \mathrm{C}\right)$.

Mechanical properties of the Langmuir monolayers were characterized by calculating surface compressional modulus $\left(\mathrm{C}^{-1}\right)$ from the measured $\pi-A$ isotherms by means of the following equation $[24,25]$ :

$$
C^{-1}=-A\left(\frac{\partial \pi}{\partial A}\right)_{T}
$$

Penetration experiments were realized on a microwell plate (glass plate with Teflon rims). The well was filled with $700 \mu \mathrm{l}$ of the required liquid (ultrapure water or the dispersion of different nanoparticles), the lipids were spread at the air/liquid interface to reach a surface pressure in the range of 10 and 35 $\mathrm{mN} / \mathrm{m}$. The change in surface pressure was recorded in a 1-h period. The recorded surface pressure-time curves were evaluated by determining the initial surface pressure $\left(\pi_{0}\right)$ and the change in surface pressure $(\Delta \pi) . \Delta \pi-\pi_{0}$ data are plotted and the measurements were evaluated based on the method developed by Salesse and colleagues [26, 27]: synergy and maximum insertion pressure values are calculated which give information on the nanoparticle-lipid interaction.

\section{AFM measurements}

The nanoparticles incorporated in the monolayer membrane were visualized by means of AFM technique after the Langmuir-Blodgett film transfer. Films were transferred at constant surface pressure in the range of 21 and $24 \mathrm{mN} / \mathrm{m}$, freshly cleaved mica sheets were used as substrates. AFM images were taken on a Nanoscope III Atomic Force Microscope from Digital Instruments with a piezo scanner capable of a deflection of $12.5 \mu \mathrm{m}$ in $x$ and $y$ directions and $3 \mu \mathrm{m}$ in $z$ direction using a tapping-type silicon tip (Veeco Nanoprobe Tips, Model RTESP, $125 \mu \mathrm{m}, 300 \mathrm{kHz}$ ).

\section{Results and discussion}

\section{Characterization of Au NPs and Au NRs}

Rod-like and spherical Au NPs of different sizes were synthesized in aqueous media in order to study different types of gold nanoparticles. TEM images (Fig. 1) showed the uniform spheres and the rods of Au. Au spheres with $10.2 \pm 0.9 \mathrm{~nm}$ (further referred as 10-nm particles) and $14.6 \pm 1.2 \mathrm{~nm}$ (further referred as $15-\mathrm{nm}$ particles) diameter and Au NRs with average aspect ratio (AR) of 2.8 (width: $7 \pm 2.1 \mathrm{~nm}$ and length: $20 \pm$ $4.5 \mathrm{~nm}$ ) were synthesized.

Cysteine and glutathione coated Au NPs were prepared in order to study the effect of the capping ligand on the incorporation of the particles into the lipid membrane. In our previous work, we have presented the detailed characterization of these biofunctionalized Au NPs: the $\mathrm{pH}$ dependent behaviour of the cysteine and glutathione modified particles [21]. Dynamic light scattering and zeta potential measurements revealed the colloid stability of Au NPs dispersion: the particles are individually dispersed above $\mathrm{pH} 6$ in case of cysteine and above $\mathrm{pH} 4$ in case of glutathione surface modification. The measured zeta potential value for unmodified particles was $-60 \mathrm{mV}$, for cysteine and glutathione modified particles were -65 and $-70 \mathrm{mV}$, respectively. Surface of the Au NRs was capped with cysteine by means of three centrifugation and washing steps. Zeta potential values of $+65 \mathrm{mV}$ and $+42 \mathrm{mV}$ were obtained for the CTAB coated and the cysteine coated AuNRs. The positive zeta potential of the nanorods is due to the adsorbed cationic surfactant coating on their surface. Earlier results showed that after the second washing step the adsorbed surfactant bilayer is also damaged beside the removal of the free, unlinked molecules. It is possible to replace surfactant molecules in this way after the first washing. The decrease in the zeta potential means that remarkable amount of the CTAB was replaced with cysteine. No complete exchange of the surfactant to amino acid can be applied due to the limited stability of the Au NRs without CTAB. 
Fig. 1 TEM image of the a 10 and $\mathbf{b} 15 \mathrm{~nm}$ cysteine coated spherical Au nanoparticles (NPs) and the $\mathbf{c}$ CTAB-coated and $\mathbf{d}$ cysteine-coated $\mathrm{Au}$ nanorods
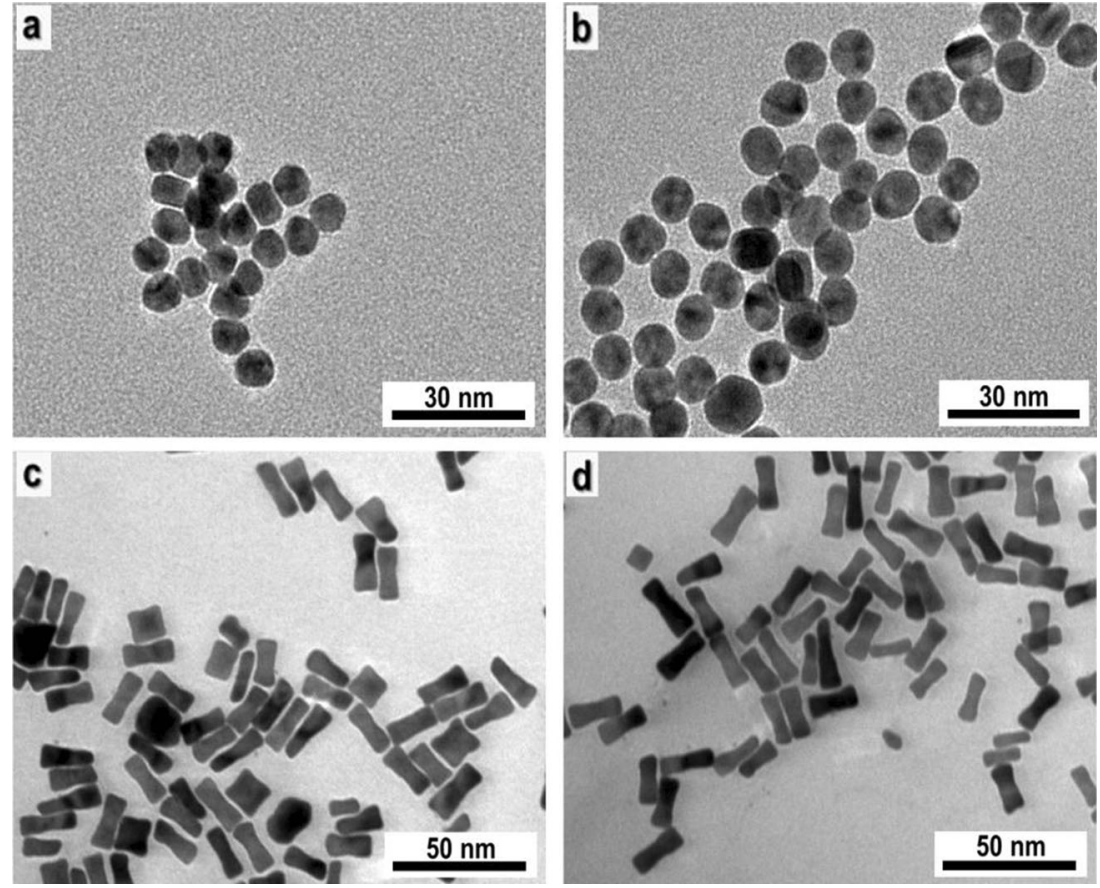

Optical properties of the samples were characterized by means of UV-VIS absorbance measurements (Fig. 2). The spectra of the bioconjugated particles of 10 and $15 \mathrm{~nm}$ average diameter show absorbance due to plasmon resonance at $518 \mathrm{~nm}$, which remained unchanged after capping. The spectrum of the rods (Fig. 2b) exhibit two absorption peaks at 520 and at $733 \mathrm{~nm}$, both of the absorption bands shifted slightly due to the surface modification of the Au NRs with cysteine. The reason for this is that the longitudinal plasmon energy is much more sensitive for the change in the local environment [28], therefore the removal of the huge amount of the surfactant and the introduction of cysteine lead to blue shift of the longitudinal plasmon absorbance peak from $733 \mathrm{~nm}$ to $723 \mathrm{~nm}$. The reason for the shift of the transverse plasmon energy is the change in the particle shape distribution. During the centrifugation of the nanorod sol a remarkable amount of the spherical and the cubic particles have been removed, which resulted in the shift of the absorption band from
$520 \mathrm{~nm}$ to $525 \mathrm{~nm}$ and the disappearance of the shoulder of this peak around $570 \mathrm{~nm}$. To support this argument we demonstrate TEM images of the Au nanorods before and after functionalization on Fig. 1. It is important to note that the absorption band, which corresponds for the longitudinal electron oscillation is in the so called biological NIR window (650-900 nm) [29], which is important for biomedical applications.

Surface pressure $(\pi)$ versus surface area $(A)$ isotherms

Model monolayer membranes of phospholipids were formed at the air/liquid interface in a Langmuir trough. The lipid films were characterized by means of surface pressure versus surface area isotherms on different subphases. The isotherm on pure water subphase shows the characteristic features for different phases, and the measured curve is in good agreement with the literature data [30].
Fig. 2 UV-Vis absorbance spectra of the aqueous dispersions of the $\mathbf{a}$ Au NPs and $\mathbf{b}$ Au NRs samples a)

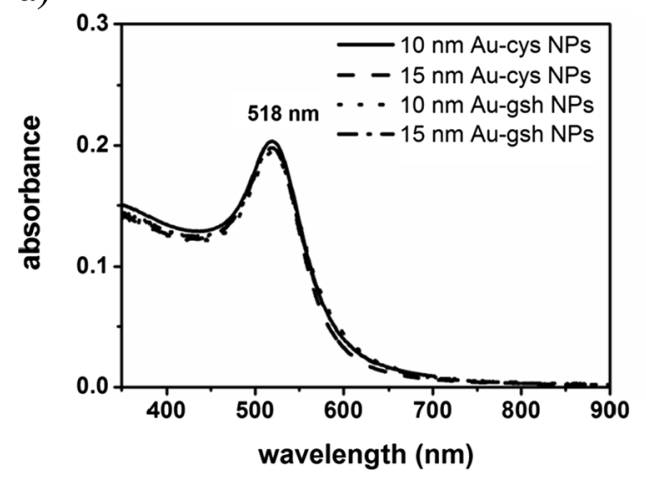

b)

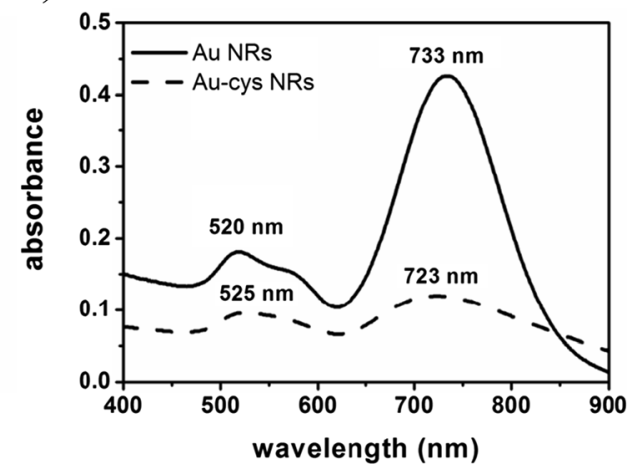


We have measured the $\pi-A$ isotherms when the Au NPs were present in the subphase. Figure 3 a shows the recorded $\pi-$ $A$ curves for cysteine coated spherical Au NPs. Isotherms on ultrapure water and nanoparticle dispersion surface were measured after $30 \mathrm{~min}$ equilibrium time. It is obvious that the particles have influence on the behaviour of the lipid monolayer: the phase transition regions are less clearly differentiated and the isotherm was shifted to larger areas assuming some nanoparticles adsorbed in the membrane in the compressed state. It is interesting that the isotherms in case of the Au-cys and Au-gsh NPs are very similar (see Fig. 3a and Fig. S1a). This can be explained with the similar properties of the two capping ligand and with the effect of the citrate residues in the dispersions. For reference we have tested the citrate reduced, unmodified nanoparticles, as well. The isotherm on Au citrate NPs sol (see Fig. S2a) shows only slight differences from the isotherm on pure water, the negatively charged citrate molecules have weaker interaction with the zwitterionic lipid molecules than cysteine or glutathione. There is no shift in the compressed region of DPPC isotherm in the presence of $\mathrm{Au}$ citrate NPs, which means that the particles were squeezed out during compression. The isotherm on cysteine-capped $\mathrm{Au}$ nanorod dispersion shows different shape (Fig. S3a). The AuNR sol has lower surface tension compared to pure water, therefore the isotherm starts at higher surface pressure value, which was also observed for surfactant coated polymeric nanoparticles [31]. The mechanical properties of the lipid films were also studied by calculating the surface compressional modulus from the $\pi-A$ isotherms (Fig. 3b; Figs. S1b,
$\mathrm{S} 2 \mathrm{~b}$ and $\mathrm{S} 3 \mathrm{~b}$ ). The surface compressional modulus gives important information about the membrane, such as the fluidity. Most of the cross-membrane processes are affected by the membrane fluidity, therefore it is important to study the effect of the nanoparticles on it [32]. The calculated curves for 10 and $15 \mathrm{~nm} \mathrm{Au-cys}$ and Au-gsh particles (by Eq. 2) show that the nanoparticles have significant effect on the compressional modulus in the surface pressure range of $5-30 \mathrm{mN} / \mathrm{m}$. At lower surface pressures $(<15 \mathrm{mN} / \mathrm{m})$ the nanoparticles decrease the compressibility, which means that the membrane becomes more fluid. In case of the surface pressure is higher than $15 \mathrm{mN} / \mathrm{m}$, the nanoparticles induce increase in the compressibility, which means a less fluid membrane. The value of $15 \mathrm{mN} / \mathrm{m}$ corresponds for the start of transition from the expanded liquid state to the condensed liquid state, at this point the compression modulus (and the fluidity) of the membrane is rapidly changing. The transition from expanded liquid state to the condensed liquid state does not appear such clearly in the presence of the nanoparticles (Fig. 3b). The compressional modulus was also calculated in case of Au-cys nanorods in the subphase (see Fig. S3b). The nanoparticles induce the shift of the phase transition to smaller surface pressure values; the model membrane becomes less fluid at smaller compactness.

\section{Interaction of nanoparticles with preformed membranes}

The interaction of biofunctionalized Au NPs with preformed model lipid membranes was characterized by means of surface
Fig. 3 a Surface pressuresurface area isotherm of DPPC on different subphases: ultrapure water, nanodispersion of 10 and $15 \mathrm{~nm}$ cysteine coated Au NPs ( $0.2 \mathrm{mM} \mathrm{Au} \mathrm{NPs} \mathrm{concentration).}$ b Surface compressional modulus of the lipid monolayers on these subphases a)
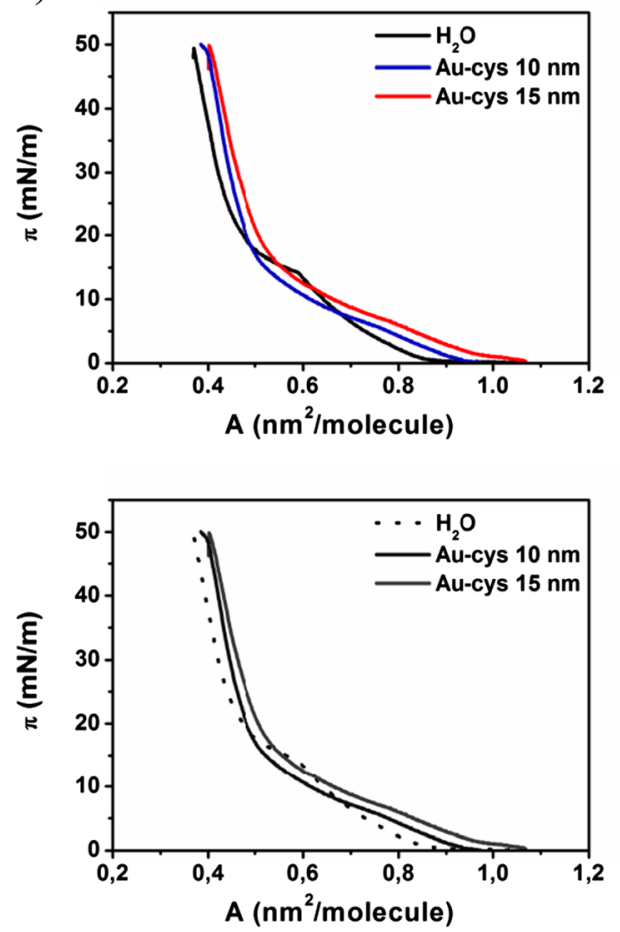

b)
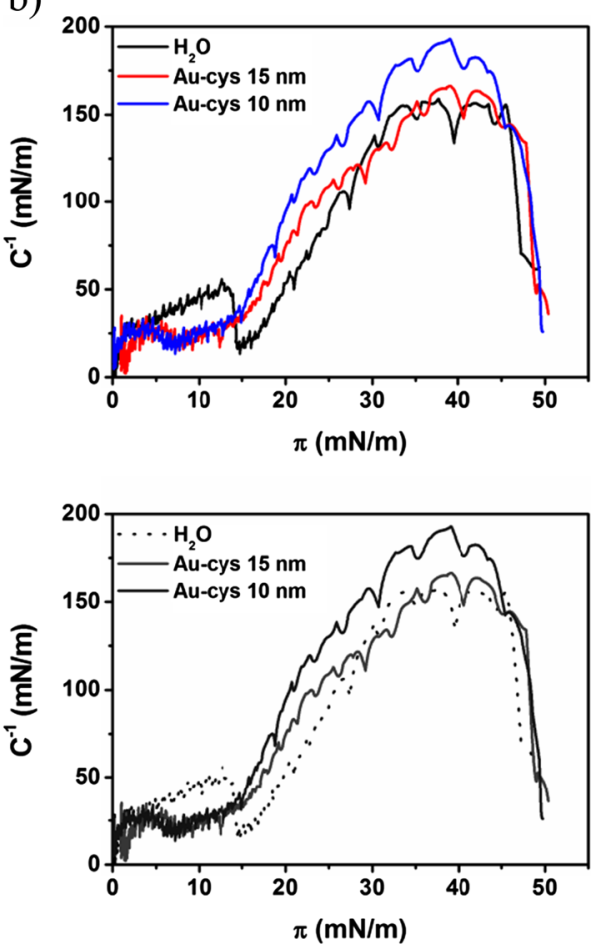


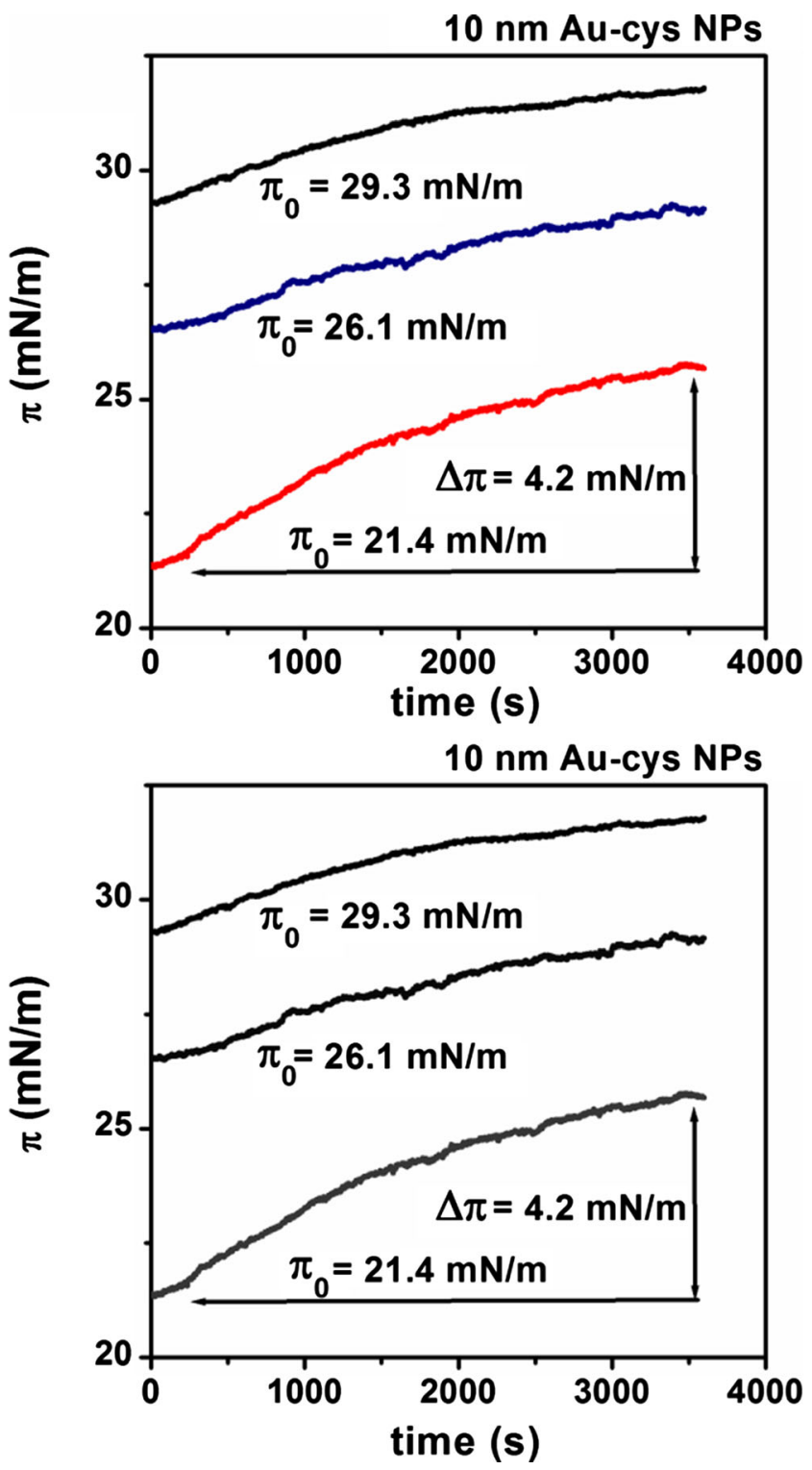

Fig. 4 Change in the surface pressure of the preformed monolayer membrane during the interaction with 10 -nm-sized Au-cys NPs at various initial surface pressures. Initial surface pressure values $\left(\pi_{0}\right)$ and the determination of the change $(\Delta \pi)$ is marked on the graph pressure measurements. The change in the surface pressure $(\Delta \pi=f(t))$ of the pure phospholipid (DPPC) model membrane was followed during the interaction with $\mathrm{Au}$ NPs in the subphase. In all, cases we have found increased surface pressure (see examples in Fig. 4 and Fig. S4) which can be explained with the interaction of the Au NPs with the lipid monolayer. Penetration of the particles into the monolayer resulted in compacter membrane and therefore increased surface pressure.

We have studied the interaction of the biofunctionalized particles with membranes of different compactness: various initial surface pressures were set and the increase in surface pressure was evaluated (Fig. 4). The plot of the surface pressure change $(\Delta \pi)$ versus the initial surface pressure $\left(\pi_{0}\right)$ gives a linear relationship. Figure 5 presents the results for the measured $\Delta \pi$ values for DPPC membranes in case of the different-sized and surface-modified nanoparticles. It can be generally concluded that the higher initial surface pressure results in smaller increase in the surface pressure. This is in good agreement with the intuitions: smaller incorporation is expected in case of compacter film.

Two important parameters can be calculated from the $\Delta \pi$ versus $\pi_{0}$ plot (Fig. 5a): maximum insertion pressure (MIP or other authors use the term critical surface pressure) and synergy. The linear fitting gives these results as the $\mathrm{x}$ tangent intercept and the slope of the line. The maximum insertion pressure gives the surface pressure value above which no incorporation can be observed. The surface pressure in living cells is in the range of $20-30 \mathrm{mN} / \mathrm{m}$ [33] depending on the type and location of the cell. Therefore, the maximum insertion pressure provides important information about the nanoparticle penetration. If the maximum insertion pressure of a given system is below $30 \mathrm{mN} / \mathrm{m}$, smaller penetration ratio in living systems is expected. The synergy relates to the affinity of the particles to the membrane. In case of synergy $>0$, positive affinity can be predicted, but if synergy $<0$ the particles have less affinity to the monolayer membrane.

We have evaluated our experimental results in this point of view (Fig. 6). In case of synergy, there is not much difference for the different particles, but it is important that positive
Fig. 5 Increase in surface pressure during $1 \mathrm{~h}$ measurement period as a function of the initial surface pressure in case of the a glutathione-coated particles and $\mathbf{b}$ the cysteine-coated $\mathrm{Au}$ nanoparticles with DPPC membrane a)

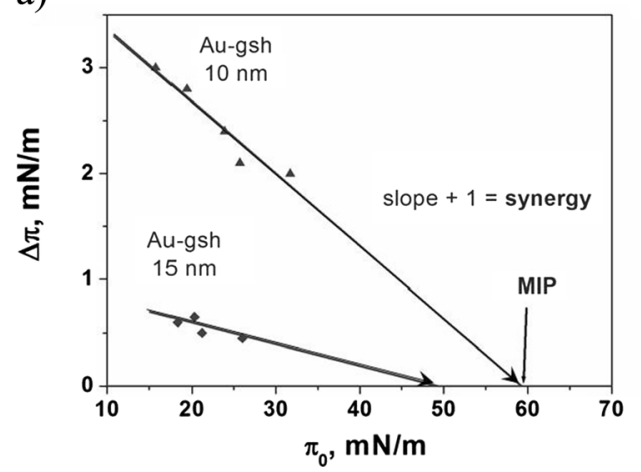

b)

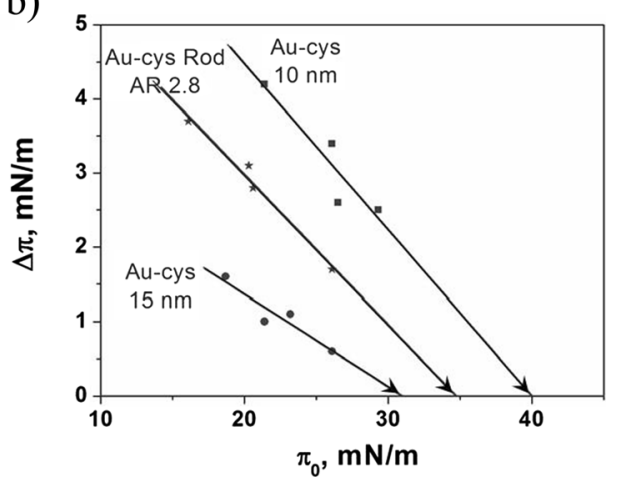


Fig. 6 Maximum insertion pressure $(M I P)$ and synergy values obtained for the different sized, shaped, surface functionalized nanoparticles with DPPC membrane a)

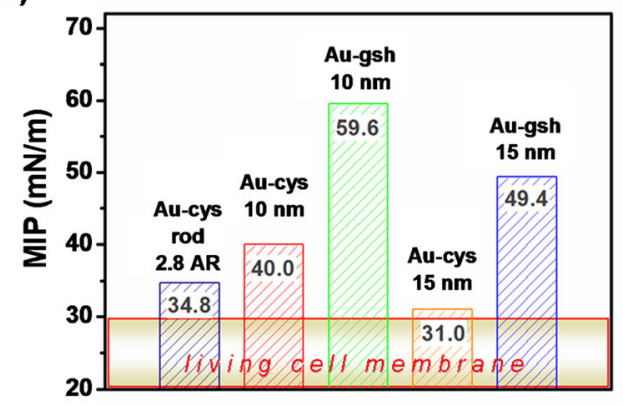

a)

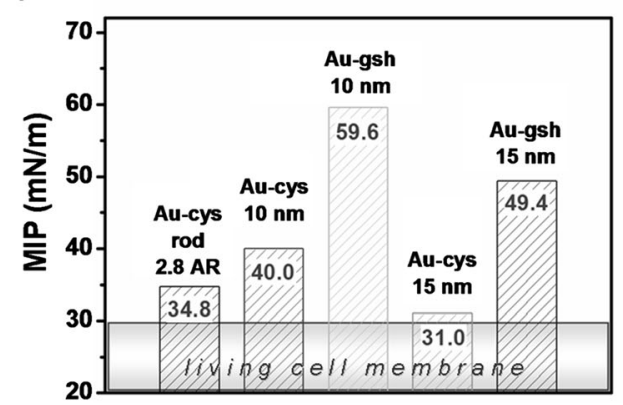

b)

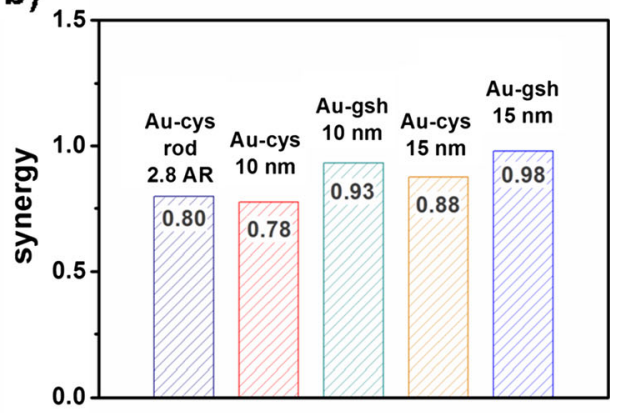

b)

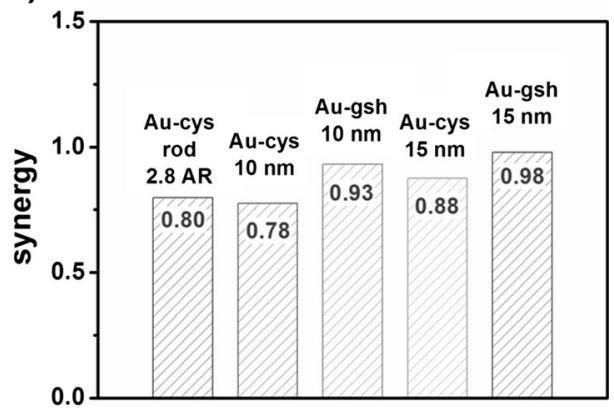

synergy was observed for all kinds of particles, which means favourable condition for incorporation. In case of MIP the results are more diverse. The gray band (in the range of 20-30 $\mathrm{mN} / \mathrm{m}$ ) represents the measured compactness in biological membranes [33]. Nanoparticle insertion can be realized if MIP is in or above this region. It can be seen that all of the studied systems reach this region; moreover, most of them have much higher MIP. The highest synergy was obtained for 10-nm-sized spherical particles with glutathione surface coating, which means that these particles can incorporate into the most compact membrane.

In our study we were focusing on the effect of different parameters influencing the interaction. In order to study particle size effects we have carried out experiments with Au NPs of 10 and $15 \mathrm{~nm}$ average diameter. The evaluation of the curves registered during penetration show that there is significant difference owing to particle size effects. We have obtained that smaller particles have higher incorporation rate compared to the larger ones. Rod-like particles have different surface properties from the spheres; therefore, it is hard to make any conclusions on the effect of particle shape. It was earlier observed, that nanorods with elongated shape have slower endocytosis, which is due to longer wrapping time of these particles [13].

In vivo experiments showed that surface properties of the particles (such as surface charge and the surface charge density) play important role in internalizing particles into cells. Nanoparticles with positive surface charge were found to cross the membrane faster than particles with negative surface, but at the same time their toxicity is much higher $[34,35]$.
In this work, cysteine and glutathione were used to cover the surface of the particles in order to characterize the role of surface properties. Both cysteine and glutathione give negative surface charge for the spherical particles, but the nanorods remained positively charged due to the incomplete removal of the cationic surfactant. It is obvious that there is significant difference in the penetration plots for different cases (Fig. 5). Cysteine-coated particles show higher incorporation rates at smaller initial surface pressures, but glutathione coated ones can incorporate into the membrane at larger membrane compactness, as well.

We have seen that the cysteine capped Au nanorod sol is slightly surface active: surface tension of the nanosol is lower than for water. To test whether the nanopartilcles alone cause increase in surface pressure we have registered the surface pressure of the nanosol without the lipid membrane in 1-h period, and we have obtained $\Delta \pi<1 \mathrm{mN} / \mathrm{m}$. This result clearly indicated that the measured increase in the surface pressure of the lipid membrane is due to the interaction with the nanoparticles and not to their surface activity alone. Surfactant-free, cysteine and glutathione coated nanoparticle dispersions have the same surface tension as water and no increase in surface tension was found in the absence of lipid membrane.

For the discussion of the interactions with the membrane it is important to consider the $\mathrm{pH}$ dependent charge of the lipid. The $\mathrm{p} K_{\mathrm{a}}$ value of the acidic group in the phospholipid head group is around 3.8-4.0 [36, 37]. In the range below $\mathrm{pH} 4$ most of the dissociable groups are protonated, therefore the molecule has a net positive charge. At $\mathrm{pH}$ above the $\mathrm{p} K_{\mathrm{a}}$, the 
lipid is zwitterionic due to the dissociated acidic and the quaternary amine group (Fig. S5).

Our spherical Au NPs are negatively charged, the isoelectic points (i.e.p.) determined by means of $\mathrm{pH}$-dependent zeta potential measurements are at $\mathrm{pH} 2.70$ and 2.30 for cysteine and glutathione capping [21]. At $\mathrm{pH}$ values smaller than the i.e.p., particles have positive surface charge, above the isoelectic point they are negatively charged (Fig. S5). In our experiments $\mathrm{pH} 7.4$ of the gold nanodispersions were used, where both type of capped Au NPs have negative surface charge and the lipids spread at the surface of the nanodispersion are zwitterionic. Considering the molecular structure of the DPPC molecule (Fig. S5), it can be seen that there are positively charged groups available for the species arriving from the subphase to interact at the membrane surface. Therefore we suppose that there is electrostatic attraction between the cysteine or glutathione molecules linked to the surface of the nanospheres and the DPPC molecules (Fig. 7).
Fig. 7 Schematic representation of the proposed interaction of cysteine functionalized Au NPs with DPPC membrane. The negatively charged cysteinecoated gold nanopartilces represent an attractive electrostatic interaction with the positively charged amine groups of the phospholipid

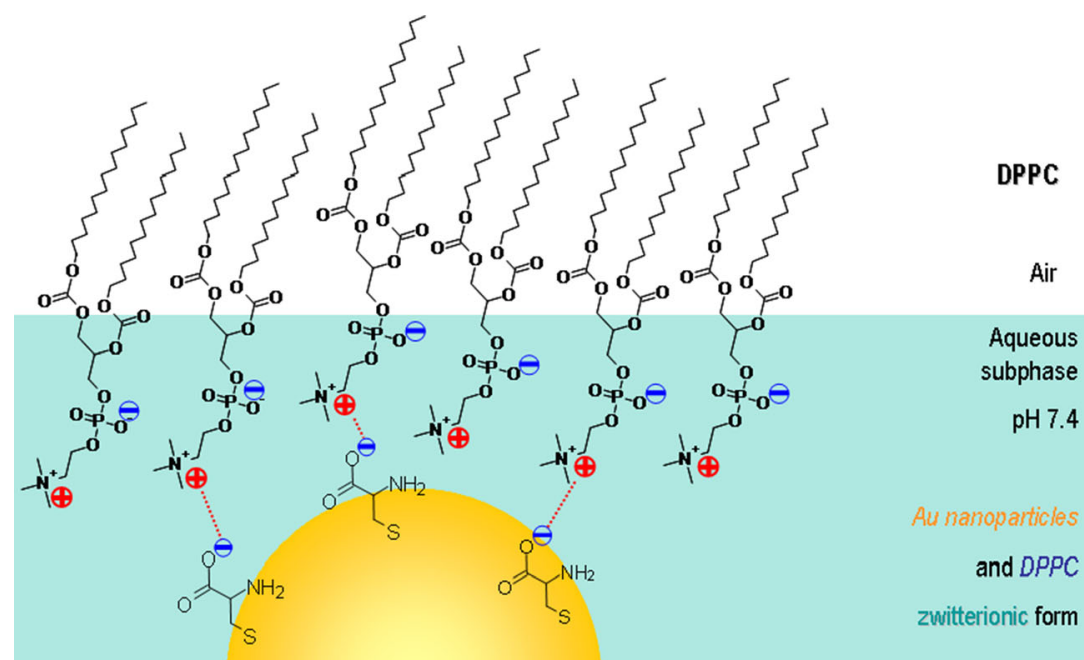

$\mathrm{Au}$

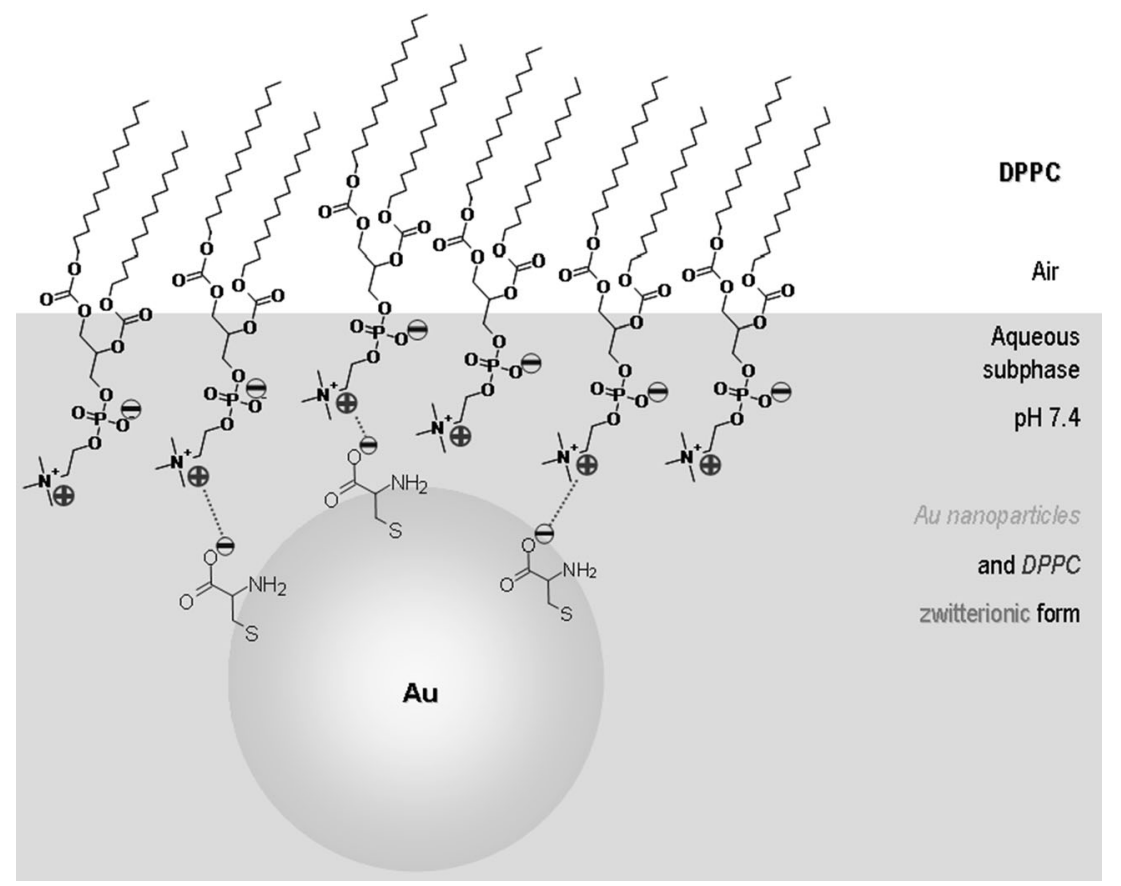


When the particles reach the surface of the lipid monolayer effect of the cations $\left(\mathrm{Na}^{+}\right.$originating from trisodium citrate) present in the nanodispersion will be significant: the cations decrease the repulsion between the phosphate group in the phospholipids and the carboxylic group in the amino acids [35]. The cysteine has one carboxyl group, while glutathione has two carboxyl groups, therefore the tripeptide has larger negative surface charge at the same $\mathrm{pH}$ value. This must be the reason for the different behaviour: the smaller negative charge is preferred for the zwitterionic head group of the lipid.

The rod shaped Au nanoparticles have positive surface charge at $\mathrm{pH} 7.4$ due to the incomplete removal of the surfactant molecules. When the Au-cys NRs reach the DPPC membrane surface there might be some repulsion between the surfactant and the amine group of the DPPC, but this repulsion is shadowed by the counter ions. The cysteine and surfactant coated nanorods can overcome this slight repulsion, and they can incorporate into the lipid layer due to the attractive electrostatic interaction between the different ionized groups (Fig. S6), and further hydrophobic interaction between the alkyl chains of the lipid and the surfactant molecules facilitate the interaction between them.

We suggest that the kinetic energy of the particles drives them to the membrane surface, but once they approached the membrane more types of interaction can influence their behaviour depending on the surface properties. In case of the negatively charged spherical particles, there is attractive electrostatic interaction between the amine group in the lipid and the carboxyl groups in the amino acids on the particles. In case of the positively charged nanorods, we suppose that beside the electrostatic forces the hydrophobic interaction plays also a key role.

\section{AFM images of solid supported membranes}

The lipid Langmuir monolayers were transferred onto solid substrates after nanoparticle incorporation so that we could visualize the nanoparticles in the membrane and obtain further information about the nanoparticle-membrane interaction. We have carried out AFM measurements to obtain the surface topographic image. Figure 8 shows the top view of a representative area of the film in case of the cysteine-coated 10-nm Au spheres, Figs. S7 and S8 present similar images for 10-nmsized glutathione coated particles and for the cysteine capped $\mathrm{Au}$ NRs. The membrane was transferred at $24 \mathrm{mN} / \mathrm{m}$ surface pressure which corresponds to the liquid compressed phase and therefore an ordered structure of DPPC [38]. The white spots on the AFM images are the Au NPs which were transferred with the membrane from the air/liquid interface. The images show small number of nanoparticles on the substrate which can be explained with the compactness of the membrane. The nanoparticle insertion leads to the decrease in the available area for phospholipids in the floating monolayer. In the biologically relevant compactness range (where we have performed our experiments), small decrease in the available area for phospholipids leads to high increase in the surface
Fig. 8 AFM image of the Langmuir-Blodgett transferred DPPC monolayer after the interaction with 10-nm-sized cysteine-coated Au nanospheres (image shows $1 \times 1 \mu \mathrm{m}$ area of the sample surface)
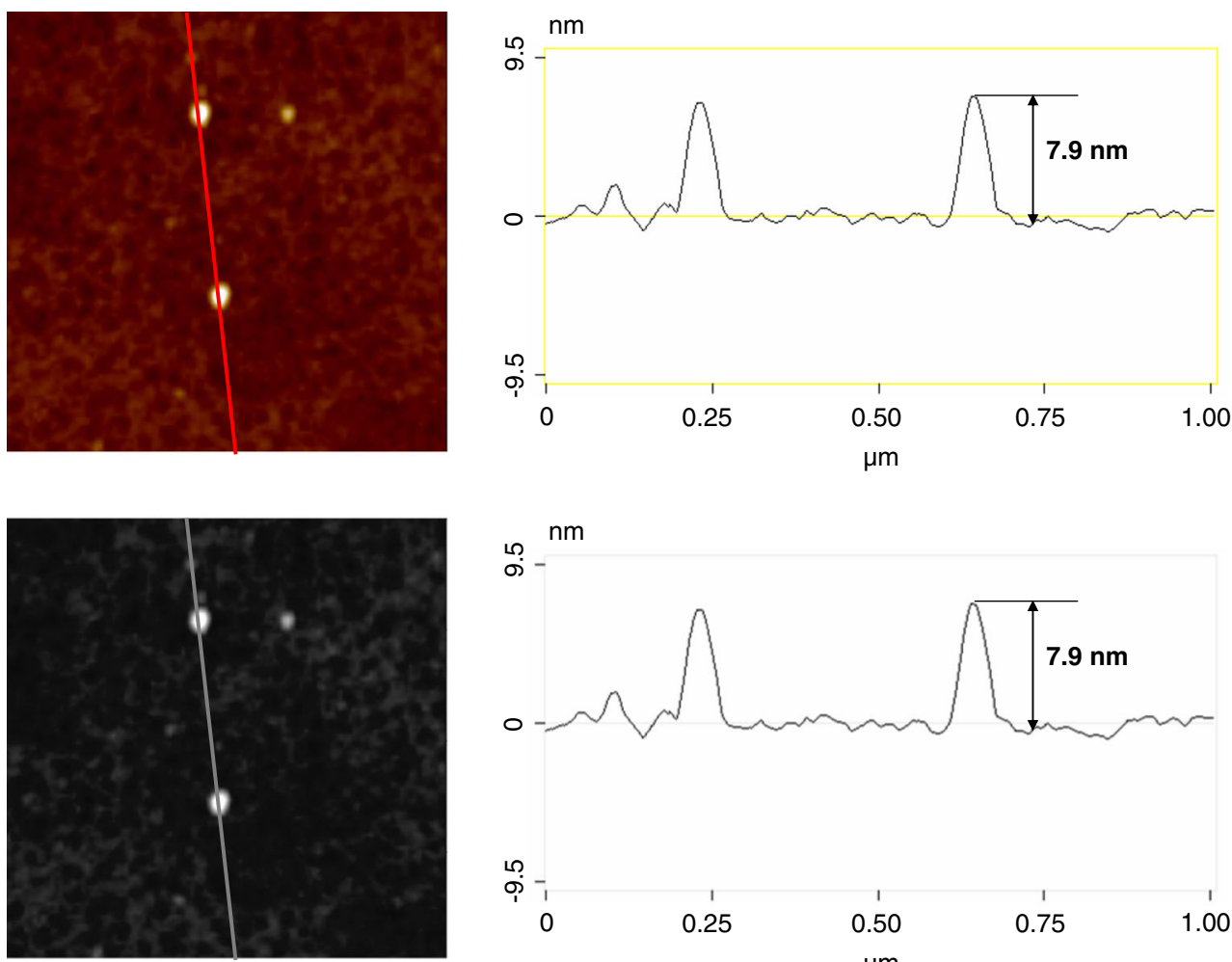
pressure, which can be judged from the steep part of the $\pi-A$ isotherms. Insertion of a small number of nanoparticles lead to the measured increase in surface pressure, therefore we cannot expect high particle density in the solid supported membrane. The magnified image of the film with $10 \mathrm{~nm}$ Au-cys NPs (Fig. 8) shows two nanoparticles, the cross-sectional analysis reveals that they are individual ones. We have measured the step height at the nanoparticles on the images to obtain information on the position of the nanoparticles. In the case of cysteine-coated particles, a typical height of $7.9 \mathrm{~nm}$ was obtained. Considering the length of a DPPC molecule to be around $2 \mathrm{~nm}$ and the average size of the Au spheres to be $10.2 \mathrm{~nm}$, it seems that the nanoparticles are incorporated in the membrane, they are not only attached to the model membrane surface. Similar situation can be assumed for the glutathione coated particles (Fig. S2). It is far more complicated to make conclusions for the Au NRs (Fig. S3), since they are not as monodispersed as the spherical particles (see Fig. 1). It can be assumed that there are nanorods both in parallel and perpendicular orientation; additionally a tilted orientation can also be possible. Although more orientation can be imagined, we suppose the particles incorporate into the membrane parallel with the alky chains, since this case means the smallest area occupied by the nanorods. The relatively wide size distribution of the rods makes it not possible to decide whether they are only attached to the surface of the membrane or they are incorporated into it. We have calculated the average particleparticle distances on the AFM images, which were $550 \mathrm{~nm}$ for $10 \mathrm{~nm}$ spherical Au-cys, $850 \mathrm{~nm}$ for $10 \mathrm{~nm}$ spherical Au-gsh and 2,360 $\mathrm{nm}$ for rod-shaped Au-cys particles.

\section{Conclusions}

Cysteine or glutathione coated spherical and rod shaped $\mathrm{Au}$ NPs interaction with lipid membranes were demonstrated here. The interaction was studied in a Langmuir trough by means of the floating monomolecular film of DPPC as model membrane. Surface pressure-surface area isotherms with nanoparticles in the subphase showed that the nanoparticles interact with the DPPC (dipalmitoylphosphatidylcholine) molecules at the interface and decrease the fluidity of the membrane at biologically relevant membrane compactness. The study of the interaction of the nanoparticles with preformed lipid monolayers showed that the particles have positive affinity to the membrane, increase in the surface pressure of the model membrane was found in every case. We have found that smaller particles have higher affinity to the membrane and cysteine coating in the particle results in higher incorporation at lower surface pressure, but the maximum insertion pressure value was higher for glutathione capped particles.
Supporting Information Supporting information is available about the $\mathrm{pH}$ dependent behaviour of the species used in this work, sketch of the interaction in case of the Au nanorods and additional AFM images.

Acknowledgments The authors are very thankful for the financial support of TÁMOP-4.2.2.A-11/1/KONV-2012-0047. This research was supported by the European Union and the State of Hungary, co-financed by the European Social Fund in the framework of TÁMOP 4.2.4. A/2-111-2012-0001 'National Excellence Program'.

\section{References}

1. Cobley CM, Chen J, Cho EC, Wang LV, Xia Y (2006) Chem Soc Rev 35:1084

2. Wei Q, Wei A (2010) In: Weissig V, D'Souza GGM (eds) Organellespecific pharmaceutical nanotechnology. John Wiley \& Sons, New Jersey, Chapter 26

3. Thanh NTK, Green LAW (2010) Nano Today 5:213

4. Hutter E, Fendler JH (2004) Adv Mater 16(19):1685-1706

5. Huang X, Jain PK, El-Sayed IH, El-Sayed MA (2007) Nanomedicine-UK 5:681

6. Pissuwan D, Valenzuela SM, Cortie MB (2006) Trends Biotechnol 24:62

7. Huang X, Jain PK, El-Sayed IH, El-Sayed MA (2008) Laser Med Sci 23:217

8. Dreaden EC, Alkilany AM, Huang X, Murphy CJ, El-Sayed MA (2012) Chem Soc Rev 41:2740

9. Choi M-R, Stanton-Maxey KJ, Levin CS, Bardhan R, Akin D, Sturgis J, Robinson JP, Bashir R, Halas NJ, Clare SE (2007) Nano Lett 7:3759

10. Ba H, Rodríguez-Fernández J, Stefani FD, Feldmann J (2010) Nano Lett 10:3006

11. Yang M, Alvarez-Puebla R, Kim HS, Aldeanueva-Potel P, LizMarzán LM, Kotov NA (2010) Nano Lett 10:4013

12. Park HH, Park H, Jamison AC, Randall Lee T (2014) Colloid Polym Sci 292:411

13. Nel AE, Mädler L, Velegol D, Xia T, Hoek EMV, Somasundaran P, Klaessig F, Castranova V, Thompson M (2009) Nat Mater 8:543

14. Verma A, Stellacci F (2010) Small 6:12

15. Lerch S, Dass M, Musyanovych A, Landfester K, Mailänder V (2013) Eur J Pharm Biopharm 84:265

16. Peetla C, Stine A, Labhasetwar V (2009) Mol Pharm 6:1264

17. Olubummo A, Schulz M, Schöps R, Kressler J, Binder WH (2014) Langmuir 30:259

18. Peetla C, Rao KS, Labhasetwar V (2009) Mol Pharm 6:1311

19. Turkevich J (1985) Gold Bull 18:86

20. Csapó E, Patakfalvi R, Hornok V, Tóth LT, Sipos Á, Szalai A, Csete M, Dékány I (2010) Colloids Surf B 98:43

21. Csapó E, Sebők D, Bohus G, Makrai Babić J, Šupljika F, Dékány I, Kallay N, Preočanin T (2014) J Dispers Sci Technol. doi:10.1080/ 01932691.2013 .817314

22. Sebők D, Csapó E, Preočanin T, Bohus G, Kallay N, Dékány I (2013) Croat Chem Acta 86:287

23. Jana NR, Gearheart L, Murphy CJ (2001) J Phys Chem B 105:4065

24. Petty MC (1996) Langmuir-Blodgett films. Cambridge University Press, Cambridge, An introduction

25. Vollhardt D, Fainerman VB (2006) Adv Colloid Interf Sci 127:83

26. Calvez P, Demers E, Boisselier E, Salesse C (2011) Langmuir 27:1373

27. Boisselier E, Calvez P, Demers E, Cantin L, Salesse C (2012) Langmuir 28:9680

28. Lee K-S, El-Sayed MA (2006) J Phys Chem B 110:19220

29. Huang X, Jain PK, El-Sayed IH, El-Sayed MA (2007) Nanomedicine 2:681 
30. Jabłonowska E, Bilewicz R (2007) Thin Solid Films 515:3962-3966

31. Peetla C, Labhasetwar V (2009) Langmuir 25:2369

32. Wanga B, Zhangb L, Baea SC, Granick S (2008) Proc Natl Acad Sci U S A 105:18171

33. Marcelja S (1974) Biochim Biophys Acta 367:165

34. Lin J, Zhang H, Chen Z, Zheng Y (2010) ACS Nano 4:5421

35. Torrano AA, Pereira AS, Oliveira ON Jr, Barros-Timmons A (2013) Colloids Surf B 108:120
36. Cevc G (1993) Phospholipids handbook. Marcel Dekker New York

37. Caro AL, Mackie AR, Gunning AP, Wilde PJ, Morris VJ, Rodríguez Nino MR, Rodríguez Patino JM (2007) Role of Electrostatic Interactions on Molecular Self-Assembly of Protein + Phospholipid Films at the Air-Water Interface In : Dickinson E, E Leser M E (eds) Food Colloids: Self-Assembly and Material Science, Royal Society of Chemistry, Cambridge, pp. 227-243

38. Zhai X, Kleijn JM (1997) Thin Solid Films 304:327 